\title{
A DIFFERENTIAL IN THE ADAMS SPECTRAL SEQUENCE
}

\author{
DANIEL S. KAHN ${ }^{1}$
}

It has been known for some time that the cohomology of the mod 2 Steenrod algebra $A$ admits squaring operations. (For example, see [4].) Since the cohomology of $A$ occurs as the $E_{2}$ term of the mod 2 Adams spectral sequence $\left\{E_{r}\left(S^{0}\right)\right\}[1]$, it is natural to ask if these squaring operations are in any way related to the structure of the spectral sequence. In $\$ 3$ we shall prove a theorem which evaluates the differential $d_{2}$ on $\alpha \cup_{1} \alpha$ if $\alpha$ is a permanent cycle.

1. We let $A$ denote the mod 2 Steenrod algebra and $B(A)$ the standard bar resolution [2, p. 32]. We let $\Delta: B(A) \rightarrow B(A) \otimes B(A)$ denote the diagonal map $[2$, p. 32] and $\rho$ the switching map $B(A) \otimes B(A)$ $\rightarrow B(A) \otimes B(A)$.

$\Delta$ and $\rho \Delta$ are chain homotopic. Any chain homotopy $S: \Delta \simeq \rho \Delta$ can be used to define a product $\cup_{1}$ in $\operatorname{Hom}_{A}\left(B(A), Z_{2}\right)$. By standard methods [4, p. 24] the $\cup_{1}$ product defines for any element $\alpha \in H^{s, r}(A)$ an element $\alpha \cup_{1} \alpha \in H^{2 s-1,2 r}(A)$. Any two chain homotopies $S_{1}$, $S_{2}: \rho \Delta \simeq \Delta$ will give the same value for $\alpha \cup_{1} \alpha$ and in particular will agree with value obtained by using the specific chain homotopy $\chi$ given on p. 36 of [2].

2. In dealing with the Adams spectral sequence, we shall use the formulation given in [1] with such additional comments as we make here. We shall use freely the definitions and notations of [1] in the remainder of this paper.

Our first observation is that a modification of the techniques of Lemma 1 on p. 46 of [3] can be used to give the following version of [1, Lemma 3.4]:

Lемма (2.1). Using the notations of $[1, p .189]$, we assume we are given a map of left $A$-complexes $\phi: D \rightarrow C$ covering $f^{*}: H^{*}(Z) \rightarrow H^{*}(X)$. Then there exists a map $g: Y_{0} \rightarrow W_{0}$ equivalent to $S^{n} f$ with $g\left(Y_{s}\right) \subset W_{s}$ (for $s \leqq k$ ) and such that $\mathrm{g}^{*}: H^{*}\left(W_{\mathrm{s}}, W_{s+1}\right) \rightarrow H^{*}\left(Y_{s}, W_{\mathrm{s}+1}\right)$ realizes $\phi$.

Note. In (2.1) and elsewhere we omit explicit mention of the dimension of skeletons to which the conclusions of (2.1) apply. For any given argument here, one may choose $n, k$ and $l$ "large enough."

Received by the editors September 8, 1967 .

${ }^{1}$ Research supported by National Science Foundation Grant GP 5591. 
In the following lemma we let $C$ be an acyclic resolution of $H^{*}\left(S^{0}\right)$ by free $A$-modules and $Y_{0} \supset Y_{1} \supset \cdots \supset Y_{k}$ a realization of $C$ with $Y_{0}$ having the same homotopy type as $S^{n}$.

Lemma (2.2). Let $\gamma \in \pi_{n+q}\left(Y_{m}\right), m+1<k$, and denote by $\bar{\gamma}$ its image in $E_{2}^{m, m+q}\left(S^{0}\right)$. Then there exists an element $\xi \in \pi_{n+q}\left(Y_{m+1}\right)$ whose image in $\pi_{n+q}\left(Y_{m}\right)$ is $2 \gamma$ and whose image in $E_{2}^{m+1, m+1+q}\left(S^{0}\right)$ is $h_{0} \bar{\gamma}$.

Proof. Let $f: S^{n+q} \rightarrow Y_{m}$ represent $\gamma$. Let $X_{0} \supset X_{1} \supset X_{2} \supset \cdots$ $\supset X_{k-m}$ be a realization for $C$ with $X_{0}$ having the same homotopy type as $S^{n+q}=S^{n+q}\left(S^{0}\right)$. Then by [1, Lemma 3.4] there exists a map $g: X_{0} \rightarrow Y_{m}$ equivalent with $f$ as a map into $Y_{m}$ and such that $g\left(X_{i}\right)$ $\subset Y_{m+i}, i \leqq k-m$.

Now (2.2) is clearly true for $\gamma$ a generator of $\pi_{0}^{S}\left(S^{0}\right)$, that is, there exists $u: S^{n+q} \rightarrow X_{1}$ such that the image of its homotopy class in $E_{2}^{1,1}\left(S^{0}\right)$ is $h_{0}$. Then the composite $g u: S^{n+q} \rightarrow Y_{m+1}$ induces the Yoneda product representation of $h_{0} \bar{\gamma}$. The homotopy class of $g u$ is the required element $\xi$.

3. We are now ready to prove the following result:

TheOREM. Let $\alpha \in H^{s, 8+p}(A) \approx E_{\tau}^{s, s+p}\left(S^{0}\right)$ be a permanent cycle in the Adams spectral sequence. Then

(i) $d_{2}\left(\alpha \cup_{1} \alpha\right)=h_{0} \alpha^{2}$ if $p$ is odd, and

(ii) $\alpha \cup_{1} \alpha$ is a permanent cycle if $p$ is even.

REMARK. Part (i) could be viewed as a generalization of Theorem 1.1 of [1]. (Recall that $h_{n} \cup_{1} h_{n}=h_{n+1}$.) Part (ii) is probably related to the fact that, for $\bar{\alpha} \in \pi_{2 k}^{S}\left(S^{0}\right)$ and $\bar{\alpha}$ of order 2 , the stable Toda bracket $\left\langle\bar{\alpha}, 2_{\iota}, \bar{\alpha}\right\rangle$ is divisible by $2[7$, p. 33] and the heuristic argument that $\alpha \cup_{1} \alpha$ is half the Massey product $\langle\alpha, 2, \alpha\rangle[2$, p. 47]- "heuristic" because we are working mod 2 . A clarification of this is likely to require analysis along the lines of Moss's theorem, which, among other things, discusses the relation of Massey products in $H^{*, *}(A)$ to Toda brackets in $\pi_{*}^{S}\left(S^{0}\right)$ [6].

Proof. Suppose $n$ is even and large relative to $p$ and $s$. Let $X_{0} \supset X_{1}$ $\supset \cdots \supset X_{k}, k>s+1$, be a realization for $B(A)$ with $X_{0}$ having the homotopy type of $S^{n}$. Set

$$
Y_{c}=\bigcup_{a+b=c} X_{a} \wedge X_{b}, \quad(K \wedge L=K \times L / K \vee L)
$$

Then $Y_{c}$ is a realization of $B(A) \otimes B(A)$ with $Y_{0}$ having the homotopy type of $S^{2 n}=S^{n} \wedge S^{n}$. Let $\tau: X_{0} \wedge X_{0}=Y_{0} \rightarrow Y_{0}$ be the switching map. Then $\tau$ is a realization of $\rho: B(A) \otimes B(A) \rightarrow B(A) \otimes B(A)$. 
Let $W_{0} \supset W_{1} \supset \cdots \supset W_{m}, \quad m \geqq 2 k$, be a realization of $B(A)$ with $W_{0}$ having the homotopy type of $S^{2 n}$. By (2.1), there exists a map $\mu: Y_{0} \rightarrow W_{0}$ realizing $\Delta$. Also, since $n$ is even, $\mu \tau$ is a realization of $\rho \Delta$.

By Lemma 3.5 of [1], there exists a homotopy $h: I \times Y_{0} \rightarrow W_{0}$ such that $h_{0}=\mu \tau, h_{1}=\mu$ and $h\left(I \times Y_{i}\right) \subset W_{i-1}$. We may assume that the base point $y \in \cap_{i} Y_{i}$ and that $h$ preserves base point. Now

$$
h^{*}: \quad H^{*}\left(W_{i-1}, W_{i}\right) \rightarrow H^{*}\left(I \times Y_{i}, \dot{I} \times Y_{i} \cup I \times U_{i+1}\right)
$$

defines a chain homotopy $S: \Delta \simeq \rho \Delta$.

Since $\alpha$ is a permanent cycle, we may choose a map $u: S^{n+p} \rightarrow X_{s}$ to represent $\alpha$. Denote by $\bar{u}$ its homotopy class in $\pi_{p+n}\left(X_{s}\right)$. It follows that the composite

$$
\begin{aligned}
\theta\left(E^{2 p+2 n+1}, S^{2 p+2 n}\right) & \\
& \rightarrow(I, \dot{I}) \times\left(S^{n+p} \wedge S^{n+p}, *\right) \stackrel{1 \times(u \wedge u)}{\longrightarrow}(I, \dot{I}) \times\left(X_{s} \wedge X_{s}, y\right) \\
& \rightarrow\left(W_{2 s-1}, W_{2 s}\right)
\end{aligned}
$$

represents $\alpha \cup_{1} \alpha$.

By [4, Lemma 22.3], $\partial_{*} \theta \in \pi_{2 p+2 n}\left(W_{2 n}\right)$ is 0 if $p$ is even, proving part (ii); $\partial_{*} \theta=2[\mu \circ(u \wedge u)]$ if $p$ is odd. Since $\mu \circ(u \wedge u)$ represents $\alpha^{2}$, it follows by Lemma (2.2) that there exists a map $f: S^{2 p+2 n} \rightarrow W_{2 s+1}$ such that $f$ represents $h_{0} \alpha^{2}$ and $f \simeq \theta \mid S^{2 p+2 n}$ in $W_{2 s}$. By using a specific homotopy, one may construct an element $\bar{\theta} \in \pi_{2 p+2 n+1}\left(W_{2 s-1}, W_{2 s+1}\right)$ such that $\partial_{*} \bar{\theta}=[f]$ and such that the image of $\bar{\theta}$ in $\pi_{2 p+2 n+1}\left(W_{2 s-1}, W_{2 s}\right)$ is $\theta$. This completes the proof.

\section{BiBliogRAPHY}

1. J. F. Adams, On the structure and applications of the Steenrod algebra, Comment. Math. Helv. 32 (1958), 180-214.

2. - On the nonexistence of elements of Hopf invariant one, Ann. of Math. (2) 72 (1960), 20-104.

3. - Stable homotopy theory, Lecture Notes in Mathematics No. 3, SpringerVerlag, Berlin, 1964.

4. A. Liulevicius, The factorization of cyclic reduced powers by secondary cohomology operations, Mem. Amer. Math. Soc., No. 42, (1962).

5. W. S. Massey, Exact couples in algebraic topology. I, II, Ann. of Math. (2) 56 (1952), 363-396.

6. M. Moss, Secondary compositions and the Adams spectral sequence, (to appear).

7. H. Toda, Composition methods in homotopy groups of spheres, Ann. of Math. Studies No. 49, Princeton Univ. Press, Princeton, N. J., 1962.

NORTHWESTERN UNIVERSITY 